Proceedings

\title{
Submucosal Exploration of EMG and Physiological Parameters in the Bladder Wall ${ }^{+}$
}

\author{
Luigi Brancato ${ }^{1, *, \neq}$, Tristan Weydts ${ }^{1, \ddagger}$, Mohammad A. Soebadi ${ }^{2}$, Dirk De Ridder ${ }^{2}$ and \\ Robert Puers 1 \\ KU Leuven, ESAT-MICAS, Leuven, Belgium \\ 2 KU Leuven, Unit of Organ Systems, Leuven, Belgium \\ * Correspondence: luigi.brancato@esat.kuleuven.be; Tel.: +32-16-325-529 \\ + Presented at the Eurosensors 2017 Conference, Paris, France, 3-6 September 2017. \\ $\ddagger$ These authors contributed equally to this work. \\ Published: 18 August 2017
}

\begin{abstract}
An implantable sensor system has been developed to explore the physiological behaviour of the bladder wall. It consists of a 3-axis digital accelerometer and a pressure sensor on the upper side of a flexible polyimide printed circuit board. On the bottom side, two metallic hooks prevent migration of the device in the bladder wall while simultaneously acting as electrodes to record the electrical activity of the detrusor muscle. The full system is inserted at the interface between the detrusor and the mucosa, preventing exposure of the transducer to the urine. This ensures protection both for inflammation of the inner bladder, and from salts deposition on the device. Three packaging layers have been applied to ensure biocompatibility of the device: Parylene-C, medical epoxy and PDMS. The device was first tested in-vitro for water diffusion. Then, the feasibility of submucosal implant was demonstrated in a cadaver pig bladder, and in-vivo recordings were successfully acquired in a rat.
\end{abstract}

Keywords: bladder wall; submucosal; acceleration; pressure; biocompatible packaging

\section{Introduction}

Urodynamic measurements, like noninvasive uroflowmetry or invasive filling cystometry, are conventional diagnostic exams performed to evaluate bladder function by measuring relevant physiological parameters [1]. Voided urine volume, urine flow, and post void residual volume can be recorded together with intravescical, detrusor and abdominal pressures. Electromyography (EMG) of the external urethral sphincter is also often used to assess micturition reflexes [2].

The above mentioned tests only allow the measurement of global bladder function, and provide no information about the local behavior of the detrusor muscle. In an attempt to expand the limited knowledge of the bladder's local motions and their relation to sensory urges [3-5], a device was fabricated to go beyond conventional cystometric measurements, and to target unexplored physiological parameters in the bladder wall.

The exploratory device, designed for acute animal tests, allows the recording of acceleration, pressure and EMG of the detrusor muscle. The transducers and the EMG electrodes are mounted on a flexible printed circuit board (PCB) to be inserted at the interface between the detrusor muscle and the bladder innermost layer, the mucosa. The submucosal implantation prevents the direct contact of the device with the urine that often results in salts deposition and inflammation of the inner bladder. 


\section{Materials and Methods}

\subsection{Device Assembly}

A flexible PCB was manufactured, including a $6 \mathrm{~mm}$ wide circular head accommodating the transducers and a $25 \mathrm{~cm}$ long flexible cable. Two commercially available off-the-shelf transducers were selected keeping in mind the requirements of small form factor and low power consumption: a 3-axis digital accelerometer (BMA-280 Bosh Sensortec) and a digital pressure sensor (MS5637 TE Connectivity). Both sensors are factory calibrated. The body temperature is well regulated in within a few degrees Celsius and therefore it is not likely to introduce significant errors in the measurement.

The BMA-280 accelerometer measures $2 \times 2 \times 0.98 \mathrm{~mm}$ and features an on-chip high pass filter that allows to correct for slow baseline drift along the three axis and therefore will not require additional calibration. The MS5637 pressure sensor is a barometric transducer designed to work in air. It is provided as a quad-flat no-leads (QFN) package measuring $3 \times 3 \times 0.9 \mathrm{~mm}$ that includes the piezo-resistive sensor die and a sensor interface integrated circuit. A protective metal cap prevents mechanical damage to the delicate electronic components. To record pressure in-vivo an insulation layer providing mechanical coupling between the sensing membrane and the bladder wall is required. Therefore, after soldering the device, the metal cap was removed and, as described in the next section, three different packaging layers were applied. The encapsulation will affect the sensor's characteristic and a re-calibration of the device will be required before implantation.

Two miniaturized stainless steel barbed hooks were produced by laser cutting, coated with a thin electroplated gold layer and soldered on the backside of the flex. The hooks act as EMG electrodes, recording the electrical activity of the detrusor muscle, and provide mechanical fixation of the sensing device in the bladder wall.

Figure 1 shows all components mounted on the sensing 'head' of the flexible PCB.

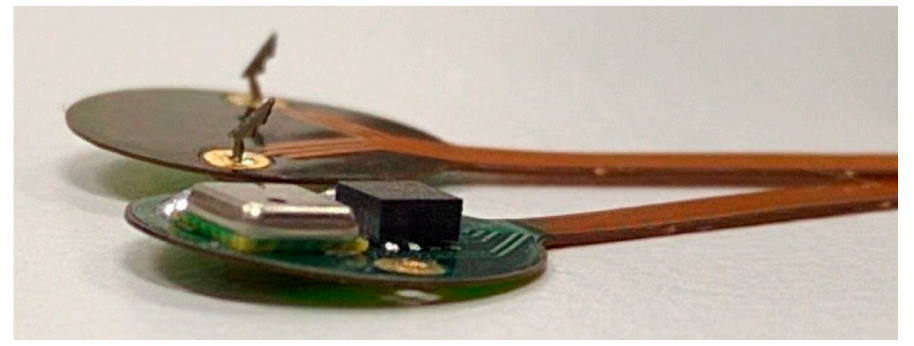

Figure 1. Bottom and top view of the device. The top contains a pressure sensor and a digital accelerometer. The bottom clearly illustrates the two protruding gold plated hook electrodes.

\subsection{Biocompatible Packaging}

To ensure biocompatibility, and to protect the electronic components from body fluids, three packaging layers were applied. First, $5 \mu \mathrm{m}$ of parylene-C were deposited by chemical vapour deposition to provide electrical insulation.

Then, a medical adhesive (EPOTEK 302-3M) was used to reinforce the solder joints on both sensors and hooks. After uncapping the pressure sensor, the same glue was applied to protect its delicate wire bonds, carefully avoiding coverage of the sensing membrane.

Finally, the complete device, with the exception of the hooks, was dip-coated in PDMS (NUSIL MED-6015). Four devices at a time can simultaneously be readout by an external circuit capable of collecting and transmitting the digital data from sensors and EMG signal over USB [6].

\section{Testing and Validation}

After assembly and packaging, the functionality of the device was proven in-vitro by soaking it in saline solution $0.9 \%$ at $37{ }^{\circ} \mathrm{C}$ for a week. The maximum current leaking through the packaging layers during device operation was measured by a sourcemeter (Keithley 2400) and found to be lower 
than $1 \mu \mathrm{A}$. To prove the feasibility of submucosal placement of the device, a fully packaged sensor was implanted in the bladder of a cadaver pig (Figure 2).

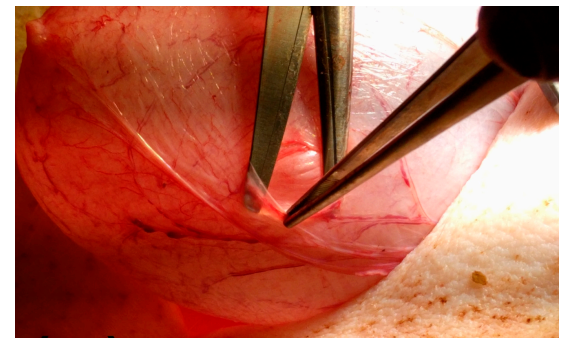

(a)

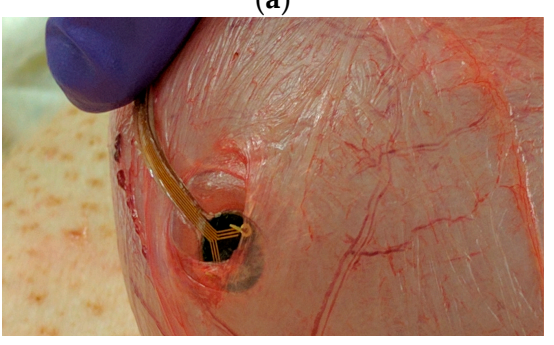

(c)

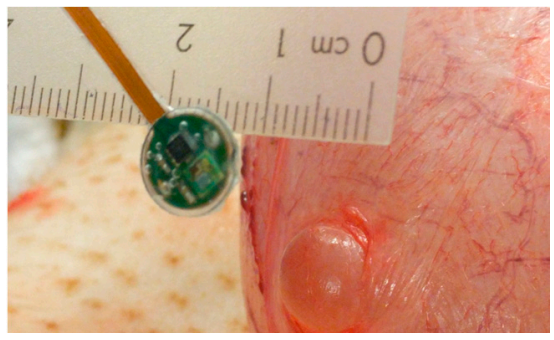

(b)

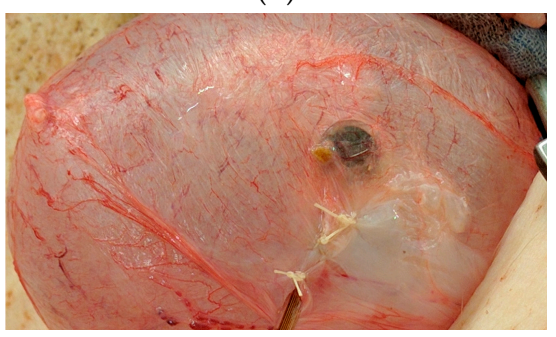

(d)

Figure 2. Submucosal insertion of the device in a pig bladder. (a) An incision is made in the detrusor muscle; (b,c) The packaged sensor is introduced; (d) The insertion hole is sutured.

An abdominal incision was carried down to gain access to the bladder. By using an intraurethral catheter the bladder was filled with saline solution. Therefore, an incision was made in the detrusor, without perforating the mucosa layer. The sensing head was then slid between the two layers with the transducers facing the organ lumen and the insertion hole was sutured. During the test the submucosal pressure and acceleration were recorded along with the reference pressure measured by the intraurethral catheter. Figure 3 shows the effect on the recorded signals of manually infusing and withdrawing saline solution three times by means of a syringe.
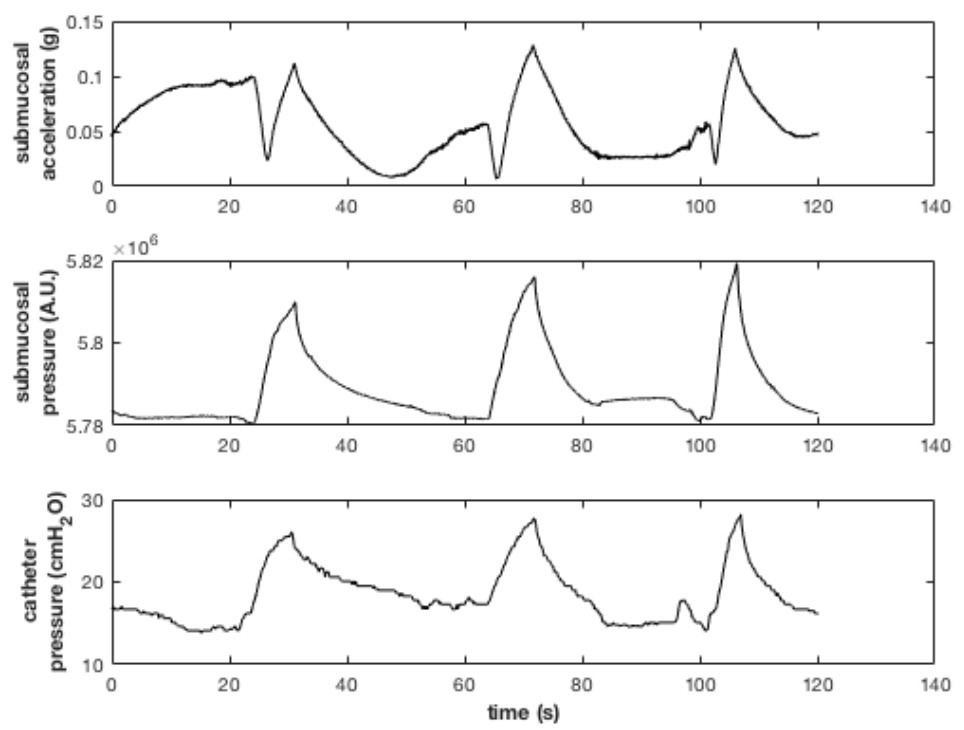

Figure 3. Signals recorded during three consecutive manual infusions of saline solution.

The submucosal signals show good agreement with the pressure recorded from the reference catheter.

Finally, an in-vivo acquisition was performed in a sedated rat (Figure 4a). As the animal's bladder was too small for implanting the device submucosally, the sensor was placed behind the organ, and kept in place with abdominal fat tissue. An implanted catheter was used to infuse saline solution at 
a controlled rate, causing the intravescical pressure to rise triggering voiding events. Figure $4 \mathrm{~b}$ shows bladder pressure, z-component of the acceleration, and EMG signal recorded during one of such voiding events.

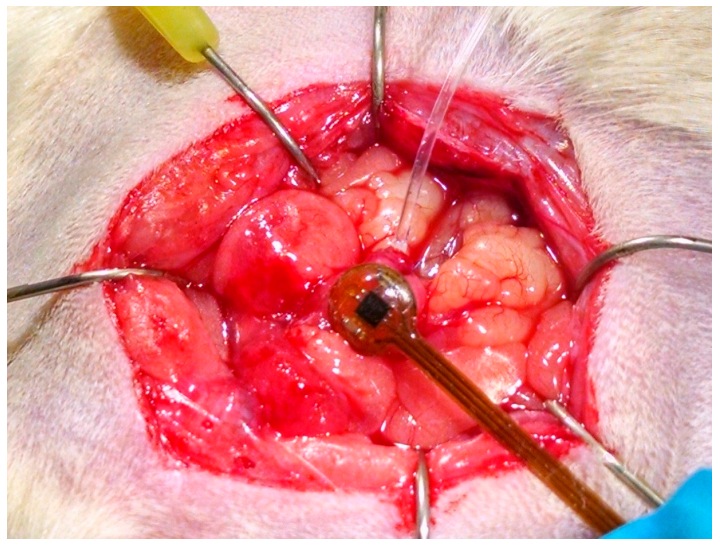

(a)

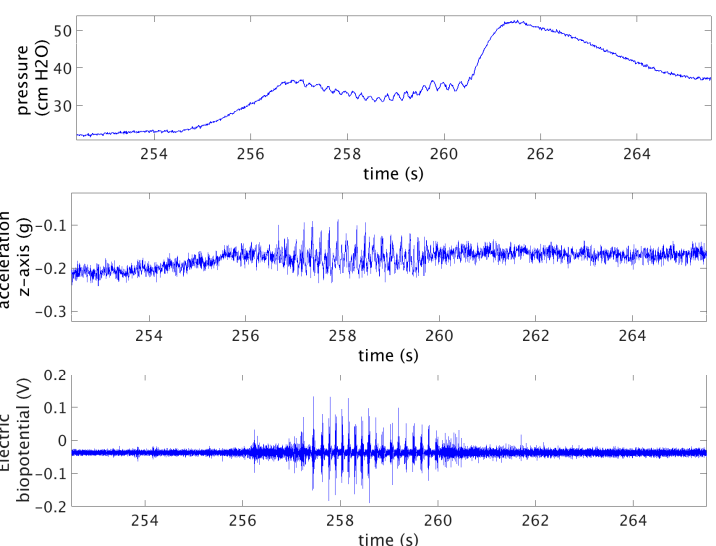

(b)

Figure 4. (a) Sensor implanted in a rat's bladder; (b) Pressure, Z-component of the acceleration and EMG recorded during a voiding event.

\section{Conclusions and Discussion}

A flexible implantable sensor system has been developed to record acceleration, pressure and EMG in the bladder wall. The device was encapsulated to ensure short term biocompatibility and to protect the electronics from the diffusion of urine and other body fluids. The device was first validated in-vitro by a soak test. A cadaver test showed the feasibility to implant the device and to record physiological parameters submucosally. Finally, an in-vivo test in a rat's bladder showed the possibility to identify and record voiding events using the proposed sensors.

This work proves, and forms the basis for the development of a more complex bladder monitoring system. It reveals an unprecedented real time measurement of a simultaneous sensing of different bladder muscle activity.

Acknowledgments: The research described in this article was performed within the ERC-2013-AG, MicroThalys, grant agreement No. 340931.

Conflicts of Interest: The authors declare no conflict of interest. The founding sponsors had no role in the design of the study; in the collection, analyses, or interpretation of data; in the writing of the manuscript, and in the decision to publish the results.

Ethical Statements: The animal experiments in this work were evaluated and approved by the Animal Ethics Committee (project number 172/2015) of the KU Leuven in compliance with all applicable national and international guidelines.

\section{References}

1. SchÌfer, W.; Abrams, P.; Liao, L.; Mattiasson, A.; Pesce, F.; Spangberg, A.; Sterling, A.M.; Zinner, N.R.; van Kerrebroeck, P. Good urodynamic practices: Uro£owmetry, filling cystometry, and pressure-flow studies. Neurourol. Urodyn. 2002, 21, 261-274.

2. Chang, H.; Havton, L.A. Differential effects of urethane and isoflurane on external urethral sphincter electromyography and cystometry in rats. Am. J. Physiol. Ren. Physiol. 2008, 295, F1248-F1253.

3. Weydts, T.; Deruyver, Y.; Brancato, L.; Dewulf, K.; Soebadi, M.A.; Weyne, E.; De Ridder, D.; Puers, R. Developing a long-term implantable system to accurately measure real-time bladder wall movements: A feasibility study in the rat. Eur. Urol. Suppl. 2016, 15, e348, doi:10.1016/S1569-9056(16)60350-0.

4. Gillespie, J.I. The autonomous bladder: A view of the origin of bladder overactivity and sensory urge. BJU Int. 2003, 93, 478-483. 
5. Nishioka, K.; Shimizu, S.; Shinohara, N.; Ito, Y.M.; Abe, T.; Maruyama, S.; Katoh, N.; Kinoshita, R.; Hashimoto, T.; Miyamoto, N.; et al. Analysis of inter- and intra-fractional partial bladder wall movement using implanted fiducial markers. Radiat. Oncol. 2017, 12, 44.

6. Weydts, T.; Brancato, L.; Soebadi, M.A.; De Ridder, D.; Puers, R. In-vivo implantable sensor system for measuring bladder wall movements. Proc. Eurosens. 2017, accepted.

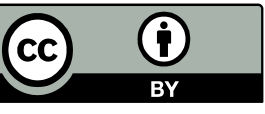

(C) 2017 by the authors. Licensee MDPI, Basel, Switzerland. This article is an open access article distributed under the terms and conditions of the Creative Commons Attribution (CC BY) license (http://creativecommons.org/licenses/by/4.0/) 